\title{
Fortune Monitor or Fortune Teller: Understanding the Connection between Interaction Patterns and Financial Status
}

\author{
Wei Pan, Nadav Aharony, Alex (Sandy) Pentland \\ MIT Media Lab \\ Cambridge, MA 02139, USA \\ Email: \{panwei,nadav, pentland\}@media.mit.edu
}

\begin{abstract}
We have deployed mobile phones to more than 100 participants in a community. In this paper, we use this unique dataset to study the correlation between users' call and Bluetooth face-to-face interaction patterns, and their financial status. We show that such correlation exists on an individual level. We find that the interaction diversity measure correlates more strongly with individual's financial status compared with other social behavior measures such as the number of contacts and length of interactions, and it is much less sensitive to personality variance. We also discuss in this paper the longlasting sociological theory that a diverse relationship leads to a more successful financial status. Our evidence tends to support a behavioral and psychological oriented theory opposite to the prevailing arguments: Social diversity exhibited by our participants are influenced by their income as well. ${ }^{1}$
\end{abstract}

Keywords-Sociology, Mobile Computing, Behavioral Science

\section{INTRODUCTION}

Recently it is discovered that interaction diversity correlates with increased wealth, as illustrated in Eagle et al [1], and it is generally believed that diversity leads to financial gain in the literature [2] [3] [4] [5].

Two immediate followup questions arise from this work: The first challenge is to study whether we can observe the same correlation at the level of individuals rather than aggregated communities. The second challenge is to better understand the causality of such correlations. The prevailing theory implies that a strong and diverse connectivity may lead to higher economic well-being, i.e. the interaction diversity is somewhat of a fortune teller. The second implication is that individuals who come from higher financial status are often those who also exhibit diverse social relationships.

We have deployed an Android-based smart phone sensing platform in a postgraduate residential community adjacent to a major research university. In addition the dataset is augmented by a comprehensive set of survey questionnaires. This study, known as the Friends and Family Study [6], has been conducted for over a year. In this study, rather than looking at aggregate area-level mobile data [1], we are interested in the individual-level relationship between one's

\footnotetext{
${ }^{1} \mathrm{~A}$ full version of this abstract is published in the proceeding for the Third IEEE Conference on Social Computing (SocialCom 2011).
}

financial status (defined as spending habits and household income in this study) and their social interaction diversity.

\section{STUDY RESUlTS}

The Friends and Family Study is split into two phases [6], the first pilot phase is conducted from Mar. 2010 to July 2010 with 55 initial participants involved. In this paper, we report the results from the second phase study (Oct. 2010 - Dec. 2010), in which we studied 85 newly added participants, and near $70 \%$ of them just arrived the university to start their graduate study since this is the start of the fall term. Because this is a residence for families, many of these participants are generally in a more advanced stage in life than that of average university students. Many had already married and have children. A large portion of the participants were already quite successful in their careers with higherthan-average salary and life styles before coming back to this post-graduate school.

\section{A. Data}

We explicitly asked two questions in the initial survey.

1) Annual household income from all sources:

2) Annual household income before coming back to this school:

We decided that the questions be categorical options rather than exact amount to reduce the feeling of privacy invasion from our participants. Therefore, we ask participants to choose one of the following options for both questions: $a$ ) Under $\$ 20,000, b) \$ 20,000-\$ 45,000, c) \$ 45,000$ - $\$ 65,000$, d) $\$ 65,000-\$ 90,000$, e) $\$ 90,000+$ and f) Prefer not to say.

There are 13 subjects who chose not to share their income status, and we exclude all these users in the analysis below.

\section{B. Analysis}

We first compute the call diversity $D_{\text {call }}(i)$ for each participant. We apply the same diversity measure as in Eagle et al. [1] The diversity $D(i)$ is defined as:

$$
D(i)=\frac{-\sum_{j=1}^{k} p_{i j} \log p_{i j}}{\log k},
$$

where $p_{i j}$ is the interaction volume between individual $i$ and $j$ divided by the total interaction volume of $i$, and $k$ 


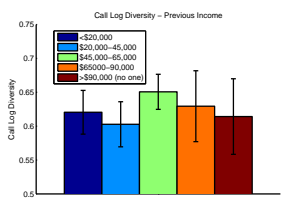

(a)

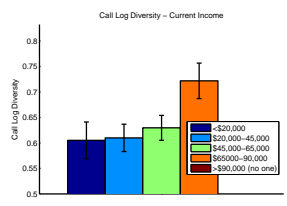

(b)

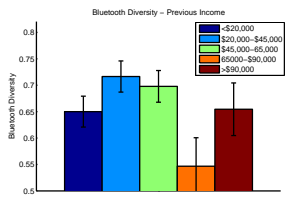

(a)

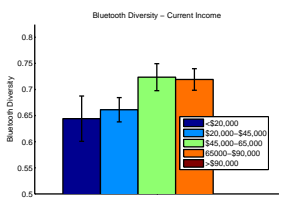

(b)
Figure 1. We show here the mean call diversity $D_{\text {call }}(i)$ and standard error for individuals in different income categories. The left plot is based on reported previous household income, and the right plot is based on reported current household income. Current household income is correlated with call diversity $(r=0.28, p=0.08)$, while previous household income is not correlated with call diversity $(r=0.003, p=0.80)$.

represents the total number of contacts. Volume is either the number of phone calls for call logs or the number of hits for Bluetooth proximity.

We illustrate the mean value and standard error for different income categories in Fig. 1. We find that there exists positive correlation between current household income and call diversity $(r=0.28, p=0.08)$. However, there is no correlation between previous estimated household income and call diversity $(r=0.003, p=0.80)$. Our observations conclude that the call diversity correlates with the current household income, but it does not correlate with previous household income.

We also look at the number of phone calls for each participant, and we discovered that there is no correlation either between the number of phone calls and current income $(r=-0.04, p=0.70)$, or between the number of phone calls and previous income ( $r=-0.05, p=0.60)$. Therefore, wealthier families do not necessarily make more phone calls, but they split their phone calls more evenly among their social ties. In addition, there is no significant correlation between the number of contacts (i.e. how many different numbers one have called) and individual's previous income $(r=0.16, p=0.30)$ or current income $(r=-0.01, p=$ $0.79)$.

We now look at the connection between income and Bluetooth face-to-face interaction diversity. Since we can not tell whether an unknown Bluetooth MAC address is a phone or other devices such as computers, the plots in Fig. 2 only include Bluetooth interactions with other participants in the study. Therefore, the interaction diversity measured here is composed of interaction only among our study participants. We illustrate the results for both previous income and current income in Fig. 2.

We notice borderline positive correlation between current household income and call diversity $(r=0.32, p=0.10)$, and we notice the correlation is much stronger within native English speakers $(r=0.53, p=0.06)$. There is no correlation between previous estimated household income and face-to-face interaction diversity $(r=-0.28, p=0.60)$.

From our data, it seems that current household income is a reasonable predictor for interactions within the community.
Figure 2. We show here the mean Bluetooth diversity $D_{\text {bluetooth }}(i)$ and its standard error for individuals in different income categories. The left plot is based on previous household income, and the right plot is based on current household income. There exists positive correlation between current household income and call diversity $(r=0.32, p=0.10)$, but there is no correlation between previous estimated household income and face-to-face interaction diversity $(r=-0.28, p=0.60)$.

Again, we discover no correlation between previous income and the interaction diversity. We also find that among all participants, those who are native English speakers tend to show stronger correlation compared with participants with other native languages rather than English. This is natural, as it takes more time for international students to improve their language skills, blend into this community and form new ties with domestic participants, as previous work pointed out [7].

In addition, we observe no correlation between overall face-to-face interaction time and income $(r=0.26, p=0.31$ for correlation with previous income and $r=0.08, p=$ 0.77 for correlation with current income). Therefore, wealthy families do not necessarily spend more time interacting with other community members.

Interestingly, we discover there exists correlation between current income and the number of face-to-face friends (i.e. the number of other community members with whom a participant has spent time) with $r=0.29, p=0.08$. However, such a relationship is not observed between previous income of the participants and the number of face-to-face friends. People with higher current income do enjoy knowing a greater number of other people in the community.

Even with a small pool, we are able to discover the connection between one's financial status (i.e. discretionary spending and income) and one's interaction diversity. Our results are well aligned with previous finding [1].

Widely used social measures such as time spent on social interactions and number of unique friends are not related to one's financial status. Our study shows that counterintuitively, wealthier individuals do not necessarily spend more or less time on meetings and calls, and neither do they necessarily have more friends or contacts.

However, it seems that the diversity measure is superior to other simpler measures such as number of phone calls or number of unique contacts in revealing one's financial status. The diversity measure is also robust to individual personality variance as described in the previous section. Our finding can benefit the mobile industry to leverage mobile data and adopt this particular diversity measure to better understand and serve their customers. 


\section{Causality}

The prevailing social theories argue that diversity brings wealth [2] [3] [4] [8] [5]. This class of causality explanations implies the following reasoning: If successful or experienced individuals are suddenly deprived of their income like many participants in this study, naturally they will continue to keep their diverse interaction behavior. Their previous experiences and success suggest that they understand and benefit from their social diversity, and their future success still relies on their continuous diversity interaction.

However, this is not the case in our study. As a matter of fact, we do not see any connection between one's immediate previous income and one's interaction pattern. We see, however, the connection between current income and interaction diversity patterns. As most participants are newly arrived students and their partners, we emphasize that their current income is largely independent of their performance, experience, previous work and opportunities from their diverse social contacts, but rather external factors that are not controllable by the individuals such as fixed stipend and limited employment opportunities for student families.

Therefore, our evidence seems to point us in the opposite direction: Individuals' social diversity is influenced by their current financial status.

Our study is very related to a recent project by Krumme et al. [9], in which researchers are investigating a large financial credit card transaction dataset to study shopping patterns of individuals. They observe that shopping diversity correlates with individuals' current financial status. By tracing users' checking accounts to establish their financial status, researchers have found that the shopping diversity (measured by entropy) for rich people is significantly higher than poor people $\left(p=10^{-4}\right)$. Krumme et al. also made use of data from the period of the recent financial downturn, and studied users who suddenly lost 20k-30k income between the year 2007 and the year 2009. It turns out that these people suddenly lost their shopping diversity by 0.05 on average, while they have not reduced their trips to shops. Their results suggest that shopping diversity is more related to current financial status and sensitive to changes in income, but overall shopping times are not sensitive to income at all.

Their observation surprisingly matches our observations on individuals who left well paid jobs to attend graduate schools. This coincidence leads us to believe that while prevailing theories are still sound, the causality mechanism is more complicated than we previously thought.

We suspect that a more behavioral and psychologically oriented mechanism plays an important role in the other direction of causality: Individuals' social diversity patterns are influenced by their financial status. We believe that as good financial status ensures people with safer and more satisfied living conditions [10], they naturally feel more confident [11] and secure in exploring new social potential [12] [13].

While in this work we provide a new perspective and some supporting evidence for this complicated causality problem, we still think that more evidence such as a more general group of subjects and a controlled long-term study is necessary to further cross examine our theory as well as other related social theories. We leave it as a future work.

\section{REFERENCES}

[1] N. Eagle, M. Macy, and R. Claxton, "Network Diversity and Economic Development," Science, vol. 328, 2010.

[2] J. Bruggeman, "Network diversity and economic development: a comment," Arxiv preprint arXiv:1011.0208, 2010.

[3] S. Page, The difference: How the power of diversity creates better groups, firms, schools, and societies. Princeton Univ Pr, 2008.

[4] M. Granovetter, "The strength of weak ties," ajs, vol. 78, no. 6, p. $1360,1973$.

[5] R. Burt, Structural holes: The social structure of competition. Harvard Univ Pr, 1995.

[6] N. Aharony, W. Pan, C. Ip, I. Khayal, and A. Pentland, "The social fMRI: Measuring, understanding and designing social mechanisms in the real world," in Proceedings of the 13th ACM international conference on Ubiquitous computing. ACM, 2011.

[7] M. Summers and S. Volet, "Students attitudes towards culturally mixed groups on international campuses: impact of participation in diverse and non-diverse groups," Studies in Higher Education, vol. 33, no. 4, pp. 357-370, 2008.

[8] M. Seidel, J. Polzer, and K. Stewart, "Friends in high places: The effects of social networks on discrimination in salary negotiations," Administrative Science Quarterly, vol. 45, no. 1, pp. 1-24, 2000.

[9] C. Krumme, "How Predictable:Patterns of Human Economic Behavior in the Wild," Master's thesis, MIT, Cambridge, MA 02139,USA, 2010.

[10] P. Frijters, J. Haisken-DeNew, and M. Shields, "Money does matter! evidence from increasing real income and life satisfaction in east germany following reunification," The American Economic Review, vol. 94, no. 3, pp. 730-740, 2004.

[11] T. Paridon, S. Carraher, and S. Carraher, "The income effect in personal shopping value, consumer selfconfidence, and information sharing (word of mouth communication) research," Academy of Marketing Studies Journal, vol. 10, no. 2, pp. 107-124, 2006.

[12] T. Clydesdale, "Family behaviors among early US baby boomers: Exploring the effects of religion and income change, 1965-1982," Soc. F., vol. 76, p. 605, 1997.

[13] S. Pong and D. Ju, "The effects of change in family structure and income on dropping out of middle and high school," Journal of Family Issues, vol. 21, no. 2, p. 147, 2000. 